\title{
Printing Method for Long Flight Distance by Laser-Induced Forward Transfer
}

\author{
Hiroyuki Suhara*, Jun Aoto and Muneaki Iwata \\ Innovation/R\&D Division, RICOH COMPANY, LTD, 2-7-1, Izumi, Ebina-shi, Kanagawa 243-0460, Japan \\ "Corresponding author's e-mail: suhara-h@jp.ricoh.com
}

\begin{abstract}
In this study, a new laser-induced forward transfer (LIFT) method that makes it possible to eject high-viscosity inks with the pressure of the ring-shaped vapor bubble (bubble ring) is presented. The flight distance should be $\geq 500 \mu \mathrm{m}$ to increase the throughput in case of industrial applications. However, in case of a long gap, the ink is scattered; therefore, good printing quality could not be obtained. We succeeded in printing high-quality droplets even for long flight distances of $500 \mu \mathrm{m}$ or more. The laser beam was shaped by the beam size and wavefront converters and then transformed into an annular beam. The experiment was conducted when the gap between the donor and receiver substrates was in the range of 200-2000 $\mu \mathrm{m}$. The landing droplet was microscopically observed to form an almost perfect circle. Our proposed system can be used in various practical applications, including QR code printing.
\end{abstract}

DOI: $10.2961 /$ jlmn.2020.02.2012

Keywords: LIFT, bubble ring, wavefront converter, droplet, flight distance, viscosity

\section{Introduction}

Digital printing technology is used not only in twodimensional (2D) printing but also in three-dimensional (3D) printing and printed electronics. Inkjet printers (IJ) [1], thermal printers (TP) [2], and electrophotography (EP) have been commercialized as digital printing technologies. Such products have already gained high reliability and customer satisfaction. IJ exhibits good performance and can be used to achieve high image quality. However, it is incompatible with high-viscosity ink due to nozzle clogging. TP and EP systems can be applied in case of high-viscosity materials; however, many restrictions are applicable with respect to the receiver materials, including the sheet shape and heat resistance.

Recently, laser-induced forward transfer (LIFT) [3-6] has attracted attention for transferring solid and liquid materials. This method can be applied when high-viscosity ink is being used because it is a nozzle-free process. LIFT also offers high flexibility in the range of receiver substrates. LIFT can facilitate applications such as 3D printing [4], printed electronics for solar cells [7], bioprinting [8], and digital printing [9].

However, the gap between the donor and receiver substrates should be kept relatively small, tens of micrometers or less, to prevent low printing quality in the LIFT system [10-12]. It is desirable that the gap is $500 \mu \mathrm{m}$ or more to increase the throughput in case of industrial applications. Furthermore, it is desirable that the gap of 1 $\mathrm{mm}$ or more can be applied in case of printing the unevenness receiver and curved surface shape. The satellite and spray are initiated when the gap is $100 \mu \mathrm{m}$ or more [13]. It has been reported that a high absorption film should be added to improve droplet quality [14-19].

On the other hand, the long-gap compatibility of LIFT has not been clarified $[19,20]$. The ink viscosities described in previously conducted studies are either undisclosed or lower than the ink viscosities considered in this study. Printing with high-viscosity inks under a flight distance of greater than $500 \mu \mathrm{m}$ is a challenge for LIFT to surpass already commercialized digital printing technology.

We have revealed that a beam profile with a central peak intensity, such as a Gaussian beam, results in ink scattering during ink ejection because the central peak intensity generates outward pressure. If the vapor pressure is directed inward, a long flight distance can be realized because of the pressure around the ink. Many LIFT studies consider Gaussian beams or do not describe beam profiles. We assumed that printing can be achieved in case of a long flight distance by controlling the beam profile. Flat top [21], Bessel [22] and Laguerre-Gaussian [23, 24] beams are renowned as particular beam profiles. There are a few studies with non-Gaussian beams in LIFT field compared to the laser processing field $[3,23]$.

In this study, we propose a novel method that generates ring-shaped vapor bubbles (bubble ring) around the ink ejection position. Further, we investigate the basic behavior and transfer characteristics associated with our method via experiments and demonstrate the possibility of high-quality printing in case of long flight distances.

\section{Experimental Methods \\ 2.1 BR-LIFT method}

Gaussian beams are generally used as the beam profile in LIFT $[19,25]$. The peak intensity of the laser coincides with the center position of the donor to be ejected. The donor position is irradiated by the pulsed laser light. Then, the absorption of the laser pulse by the donor substrate causes the evaporation of a small fraction of the donor substrate, resulting in vapor bubbles. These bubbles expand rapidly at extremely high pressure, thereby pushing out the donor volume. Thus, the donor is ejected and deposited by the receiver substrate after flight. The vapor pressure acts from the axis on which the donor is ejected to the outside in this method [25].

Next, our new LIFT method is described. In this method, the laser axis coincides with the ink ejection position. The 
donor position is irradiated by the pulsed laser light in a ring shape. The ring-shaped vapor bubble (hereinafter referred to as "bubble ring" or "BR") is generated in the boundary area between the transparent substrate and the donor substrate, as shown in Fig. 1 (a). This BR creates an inward pressure toward the axis, as shown in Fig. 1 (b). This phenomenon is consistent with the lateral flow observed in the central zone when two expanding bubbles are slightly separated [39].

Then, BR expands rapidly at extremely high pressure, driving the wrapped donor volume. Finally, the donor is pushed out; it flies and lands on the receiver, as shown in Fig. 1 (c). Hereinafter, the LIFT of ejecting the donor based on the pressure of the BR is referred to as BR-LIFT.

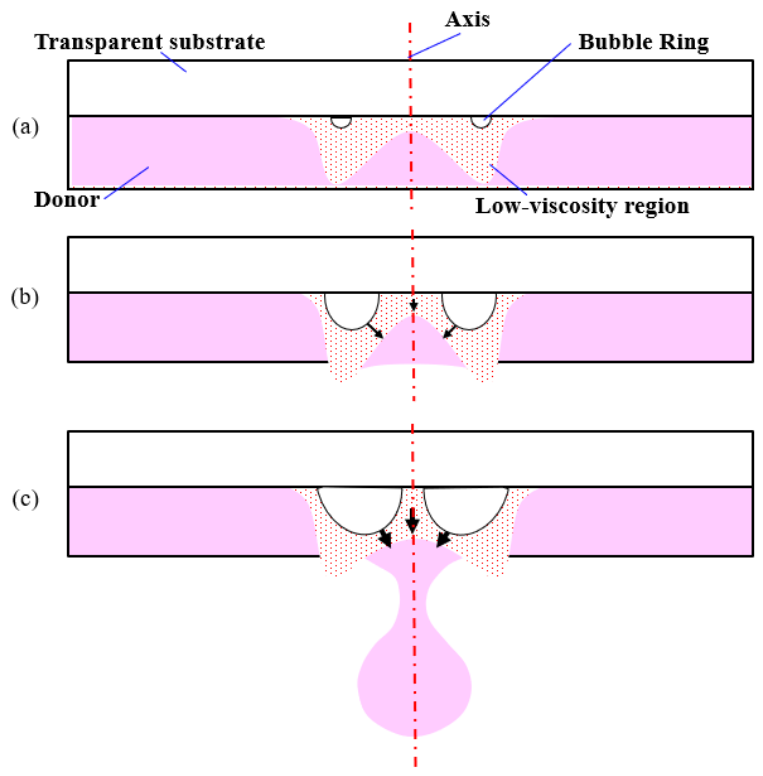

Fig. 1. BR-LIFT process: (a) the bubble ring is generated, (b) the bubble ring expands at a very high pressure, driving the wrapped donor volume, and (c) the donor is pushed out.

Forward pressure acts on the center of two beams when the irradiation interval between the beams is $100 \mu \mathrm{m}$ or less [39]. In addition, there is a report stating that forward pressure acts along the center axis of a toroidal bubble, which is obtained via the conversion of an initially spherical bubble using a counter jet [40]. This report indicates that BR is responsible for the ejection of the central liquid, although the conditions, such as the liquid free-surface and laser irradiation with a high-NA objective lens, differ from those considered in our study. A simulation study conducted based on Bernoulli's equation also reported that forward pressure acts on the central axis of a toroidal bubble [41].

The model presented in Fig. 1 is applicable when the donor substrate is a thin film and the laser beam is irradiated so that the beam diameter is almost constant in the depth direction of the thin film. Further, the Rayleigh length [38] is sufficiently larger than the film thickness. In case of a propagating Gaussian beam, the Rayleigh length $Z_{R}$ is given by

$Z_{R}=\frac{\pi \omega_{0}^{2}}{\lambda}$ where $\lambda$ is the wavelength and $\omega_{0}$ is the radius of the beam waist [38]. The beam radius $\omega(z)$ is given by

$\omega(z)=\omega_{0} \sqrt{1+\left(\frac{z}{z_{R}}\right)^{2}}$

where $\mathrm{z}$ is the distance from the beam waist point. Therefore, when the film thickness is $T_{h}$, the necessary conditions are $Z_{R} \gg T_{h}$ and $\omega\left(T_{h}\right) / \omega_{0} \cong 1$.

\subsection{System setup}

The light source used in BR-LIFT must exhibit a highquality beam profile and high power. Therefore, a nanosecond-pulsed fiber laser based on the master oscillator power amplifier (MOPA) laser system is used as the laser light source unit with a wavelength of $1064 \mathrm{~nm}$. The prototype MOPA laser comprises a two-stage preamplifier and a main amplifier. The output energy of the pulsed laser becomes $0.2 \mathrm{~mJ}$ when the repetition rate is $50 \mathrm{kHz}$. The pulsed laser passes through a spatial isolator, a quarter-wave plate, a collimator lens, and an acousto-optic deflector (AOM). The repetition rate of the pulsed laser is controlled by the AOM. The laser then passes through a nonlinear optical crystal, resulting in second-harmonic generation (SHG). SHG acts to convert a wavelength of $1064 \mathrm{~nm}$ to a wavelength of $532 \mathrm{~nm}$.

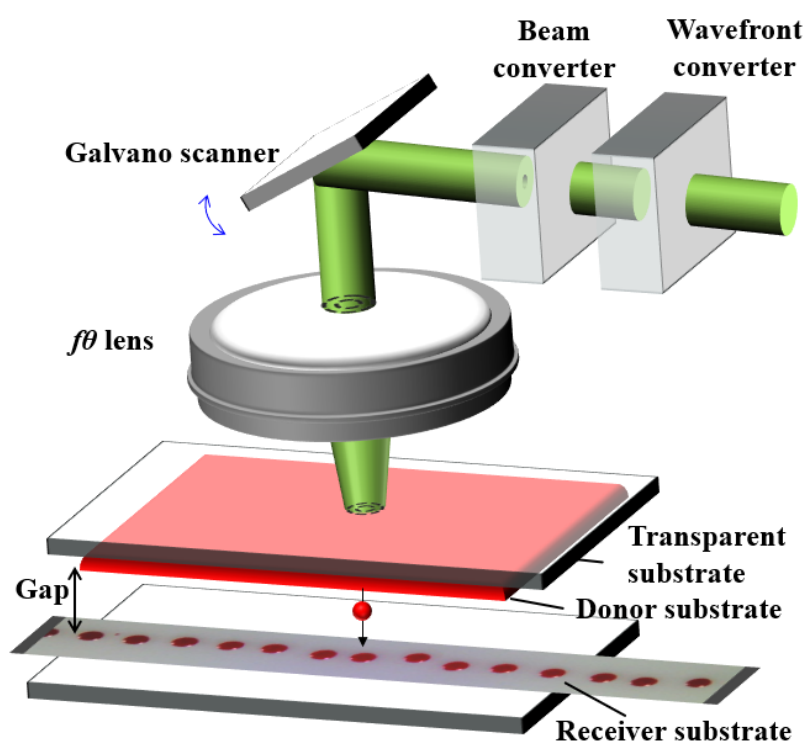

Fig. 2 Schematic of the BR-LIFT setup.

Figure 2 shows an optical system in which a pulsed laser with a wavelength of $532 \mathrm{~nm}$ irradiates the donor substrate. The laser beam is shaped by the beam size and wavefront converters [21] and then transformed into an annular beam.

Adjusting the vertical-to-horizontal diameter ratio is essential for high-quality beam generation. Because the beam size converter comprises double prisms, the beam size can be varied along the $\mathrm{x}$-axis by changing the angle of the prism with respect to the incident beam [21]. The double prism method can magnify the beam size along one dimension, converting an elliptical laser beam into a circular beam. The wavefront converter is presented in Section 2.3. The BR is generated by passing the shaped laser beam 
through a beam converter that comprises a spatial light modulator (SLM) as shown in Fig.3. The SLM transforms the beam into the desired beam profile. Subsequently, the beam passes through a mirror and other optical elements; then, it is deflected by a galvano scanner. Finally, it is focused on a donor substrate by an $f \theta$ lens. The donor material is applied to a transparent substrate. A receiver is placed in parallel on the back side of the donor substrate on an adjustable stage.

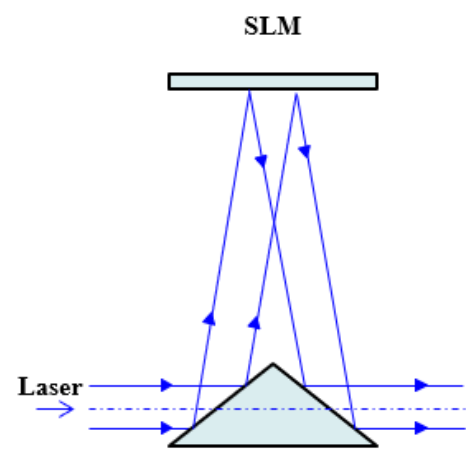

Prism mirror

Fig. 3 Beam converter: The laser beam is reflected by the prism mirror and reaches a spatial light modulator (SLM). The laser beam translated by the SLM is reflected by the prism mirror again and travels in the incident beam direction.

Several methods are available for generating an annular beam, including a geometrical optical method using lenses and a wave-optical method using a diffractive element. A Gaussian beam can be converted to a particular beam by various methods involving the usage of an axicon lens, a diffractive optical element (DOE), and an SLM. The generation of an annular beam using an axicon lens is described as follows. Based on the apex angle $\alpha$ of the axicon lens, the ring radius $R_{0}$ of the annual beam can be obtained as

$R_{0}=(n-1) \alpha F$,

where $n$ is the refractive index and $F$ is the focal length [26, 27]. The apex angle $\alpha$ or the focal length $F$ may be changed to decide $R_{0} ; R_{0}$ can be determined by changing the distance between the axicon lens and the condenser lens. SLM can be used to generate a wavefront transformed into an annular beam [28].

A Laguerre-Gaussian beam can be generated using a phase plate or cylindrical lens [29, 30]. Figure 4 shows the calculation results of the Fraunhofer diffraction image when the axicon wavefront of the apex angle $\alpha$ is superimposed on the topological charge $L$ of the Laguerre-Gaussian beam [23, 31].

The topological charge $L$ expresses a twist number, which is in one wavelength. Because the topological charge $L$ is an integer number, only a discrete ring diameter can be obtained. The ring diameter measured at $L=1$ and $\alpha=0$ was $45 \mu \mathrm{m}$, and the ring diameter at $L=2$ and $\alpha=0$ was $55 \mu \mathrm{m}$. It is difficult to obtain other ring diameters using only the Laguerre-Gaussian beam.

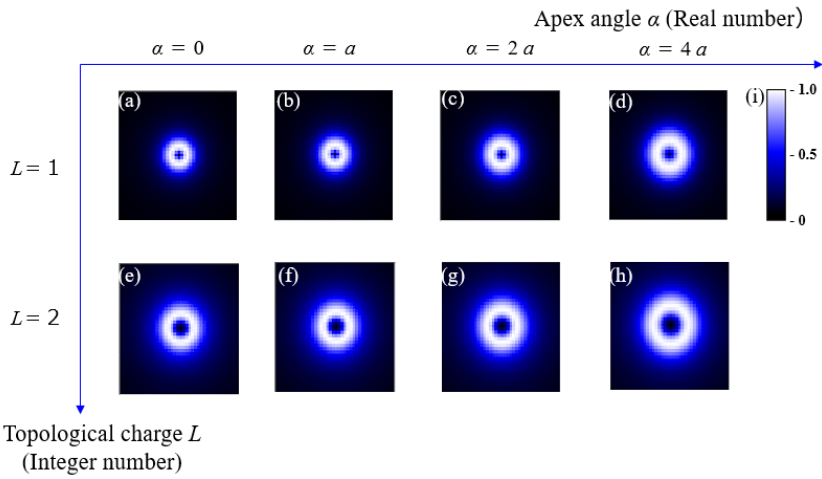

Fig. 4 Fraunhofer diffraction images generated at the focal position when the axicon wavefront of the apex angle $\alpha$ is superimposed on the wavefront with different topological charges $L$ : (a)-(d) $L=1$ and (e)-(h) $L=2$. Panel (i) is the intensity scale bar.

In contrast, the ring diameter can be freely selected because $\alpha$ is a real number. The row direction in Fig. 4 presents the calculation results obtained when $\alpha=0, a, 2 a$, and $4 a$. The diameter increases with the increasing $\alpha$. When $\alpha=0$, the axicon wavefront was not considered. The ring diameter was $125 \mu \mathrm{m}$ when $\alpha=4 a$ and $L=1$, whereas the ring diameter was $135 \mu \mathrm{m}$ when $\alpha=4 a$ and $L=2$. The ring diameter can be arbitrarily established by superimposing the conical wavefront. In addition, the ratio of the inner diameter to the outer diameter can be changed by combining $L$ and $\alpha$. Therefore, the optimum beam profile can be selected for the donor material.

An ideal beam profile is an intensity distribution in which the periphery has the maximum value and the intensity decreases toward the center. Thus, it is considerably different from that observed when using a Gaussian beam.

\subsection{Wavefront converter}

The wavefront quality of the beam entering the beam converter is influential with respect to the effectiveness of the BR-LIFT process. Even when an ideal beam is observed immediately after being emitted from the laser light source, wavefront aberrations, such as astigmatism, may occur because of the thermal lens effect in the SHG crystal.

Figure 5 shows the wavefront converter device in which double cylindrical lenses are used [21]. In this device, the first and second lenses are convex cylindrical lenses. In case of a cylindrical lens, the focal points are different between the $x$-axis and the $y$-axis direction. Here, the distance between the principal point of the first lens and the principal point of the second lens is defined as the distance $L_{f}$. The focal position of the $x$-axis and $y$-axis coincides when $L_{f}$ is twice the focal length $f$ of the lenses $(2 f x)$. The focal position along the $x$-axis is shifted toward the laser source when $L_{f}$ is greater than $2 f x$. However, the focal position along the $\mathrm{x}$-axis is shifted toward the workpiece when $L_{f}$ is less than $2 f x$. Under any condition, the focal position along the $y$-axis is fixed. Therefore, using this device, the focal position can be shifted only along the $x$-axis.

The effect of astigmatism correction can be confirmed using a wavefront sensor. Thus, all the Zernike polynomials of $\mathrm{n} \leq 2$ can be eliminated by adopting the cylindrical lens 
method [21]. Therefore, astigmatism correction is one of the most crucial factors that have to be eliminated for successful implementation of the BR-LIFT process.

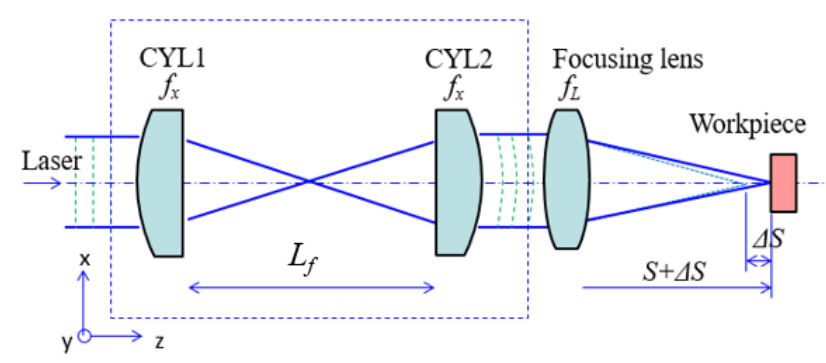

Fig. 5 Wavefront converter using double cylindrical lenses.

\subsection{Donor substrate}

The viscosity of the donor material was adjusted to the desired value by appropriately adding a viscosity modifier (UV DG reducer, T \& K TOKA) to the UV curable ink (UV Core Type A, T \& K TOKA). The UV curable ink comprised an acrylic photosensitive polymer and Pigment Red 57.1, and the viscosity modifier comprised an acrylic photosensitive monomer. The viscosity of the adjusted ink was measured using a rheometer (HAAKE RheoStress600, Thermo Fisher Scientific). The ink film was formed on the glass substrate with a wire bar coater. The wire bar coater is a bar in which wires having different thicknesses are wound. The ink film thickness can be controlled based on the amount of liquid retained by the grooves formed between different wires. The film thickness was calculated from the ink weight, coating area, and ink density. The donor material contained no component that evaporates at atmospheric pressure and room temperature in order to ensure long-term usability (even after several months). Therefore, the droplet volume remained almost unchanged over time.

\section{Results and Discussion}

Experiments were conducted to investigate the basic behavior of the BR-LIFT process. Under all the experimental conditions, the light source was a nanosecond laser with a wavelength of $532 \mathrm{~nm}$, the incident beam diameter was $3 \mathrm{~mm}$, and the focal length of the $f \theta$ lens was $100 \mathrm{~mm}$. These conditions correspond to a beam diameter of $33 \mu \mathrm{m}$ with the Gaussian beam at the focal position. By substituting the most severe $\omega_{0}(16.5 \mu \mathrm{m})$ in this experiment into Equations 1 and $2, Z_{R}$ and $\omega\left(T_{h}\right)$ were determined as $1.6 \mathrm{~mm}$ and $16.52 \mu \mathrm{m}$, respectively. Therefore, $\omega\left(T_{h}\right) / \omega_{0}=$ 1.001 , indicating that the beam diameter is almost constant in the depth direction of the thin film.

First, experiments were conducted to investigate the transfer characteristics. Next, we studied the dynamics of droplets in case of long flight distances. Finally, QR code printing is described.

\subsection{Transfer characteristics}

Figure 6 presents the experimental results obtained for magenta ink with a viscosity of $4 \mathrm{~Pa} \cdot \mathrm{s}$ and a surface tension of $29.3 \mathrm{mN} / \mathrm{m}$ [32-34]. The ink donor substrate was $20-\mu \mathrm{m}$ thick. The receiver substrate was a photo paper, and the gap was set to $500 \mu \mathrm{m}$. One-shot exposure was performed at a scanning speed of $100 \mathrm{~mm} / \mathrm{s}$ at $200-\mu \mathrm{m}$ intervals.

In Fig. 6(b), a ring diameter of $110 \mu \mathrm{m}$ and a pulse energy of $32 \mu \mathrm{J}$ was considered to perform the experiment. Droplets were formed with high reproducibility, as shown in Fig. 6(b). Based on the analysis, a dot size accuracy of 3\% or less was obtained. This dot accuracy is comparable to that observed in case of electrophotography. Thus, this method exhibits a high-quality printing capability comparable to that of electrophotography.

In contrast, Fig. 6(a) shows the measurement result obtained using a Gaussian beam with a beam diameter of 33 $\mu \mathrm{m}$ and the optimum pulse energy of $10 \mu \mathrm{J}$. Although the irradiation interval $(200 \mu \mathrm{m})$ was that of BR-LIFT, the droplets with the Gaussian beam were almost scattered and their landing position was not confirmed.

The scattering phenomenon of the droplet was not eliminated when the pulse energy was changed under the aforementioned conditions. Furthermore, droplets without scattering could not be formed even when the beam diameter was changed over $30-100 \mu \mathrm{m}$. Thus, high-quality droplets (Fig. 6(b)) could not be obtained even when the beam diameter and pulse energy were arbitrarily changed.

It has been reported that a Gaussian beam decomposed the donor film into many micrometer-scale fragments, without formation of a jet, at any pulse energies [23]. According to the time-resolved imaging observed using a high-speed camera, conventional LIFT with a Gaussian beam mostly scatters high-viscosity ink without formations of a jet [23, 36-37].

A droplet pattern with a Gaussian beam can be optimally obtained in case of high-viscosity ink using a short gap. Further, a dot-shaped pattern was generated only with a gap of $0.1 \mathrm{~mm}$ from among gaps of $0.1,0.2$, and $0.5 \mathrm{~mm}$ [37].

(a)

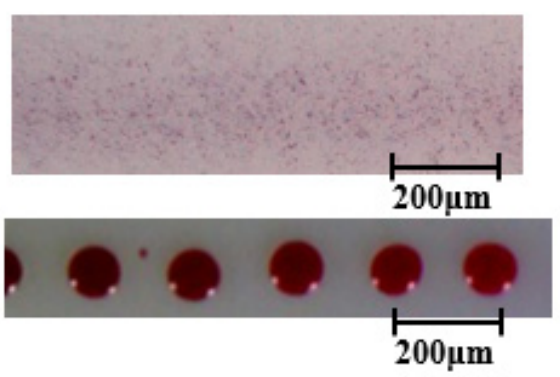

Fig. 6 Printing results obtained in a line at an interval of $200 \mu \mathrm{m}$ using a galvano scanner. (a) droplets for LIFT with a Gaussian beam, (b) droplets for BR-LIFT.

We analyzed why LIFT with the Gaussian beam and BR-LIFT provided different results. BR creates an inward pressure toward the axis, as described in Section 2.1. The low-viscosity region near BR is assumed to result in higherquality flight. Andrade's equation has been proposed to describe the temperature dependence of viscosity:

$\eta=B \exp (E / R T)$ 
where $\eta$ is the dynamic viscosity, $T$ is the absolute temperature, $R$ is the Boltzmann constant, $B$ is the proportional constant, and $\mathrm{E}$ is the activation energy.

When the donor material complies with Andrade's equation, the viscosity of the donor must decrease because of the temperature rise associated with laser irradiation. The temperature of the donor increases because of heat accumulation via laser irradiation. Thus, a low-viscosity region is created around the BR. Heat accumulation must reduce the viscosity of the donor if the donor material complies with Andrade's equation. The low viscosity inside the BR results in a considerable inward pressure gradient toward the axis. The low-viscosity liquid wraps around the high-viscosity liquid. Therefore, we believe that highquality flying can be achieved even for high-viscosity materials

Figure 7 shows the measurement results with respect to the relation between the pulse energy and droplet diameter when the ring diameter was $115 \mu \mathrm{m}$. The droplet diameter remained almost constant as the pulse energy increased by $32 \%$ (from $34.6 \mu \mathrm{J}$ to $45.8 \mu \mathrm{J}$ ). In addition, no droplets were generated at pulse energies smaller than $34.6 \mu \mathrm{J}$. Thus, reducing the pulse energy to the threshold energy did not produce small droplets. These results indicate that the droplet diameter remains constant regardless of the irradiation energy. This is one of the features of BR-LIFT obtained from the experimental results.

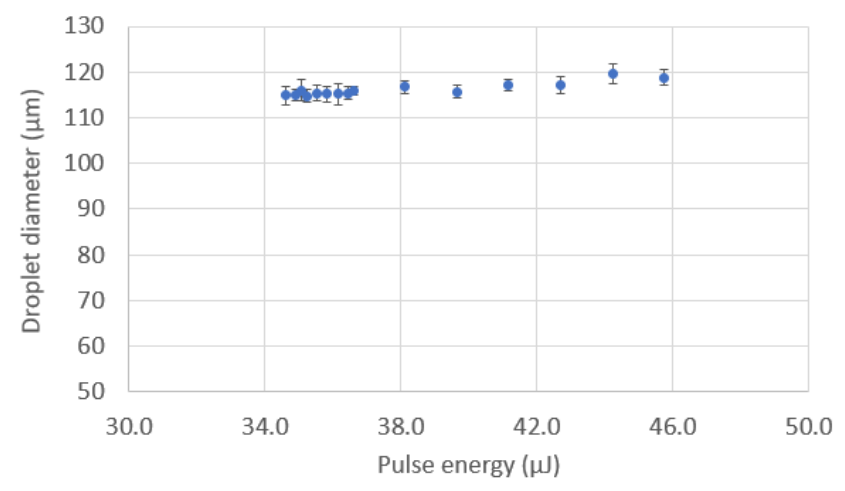

Fig. 7 Measurement results of the relation between the pulse energy and droplet diameter.

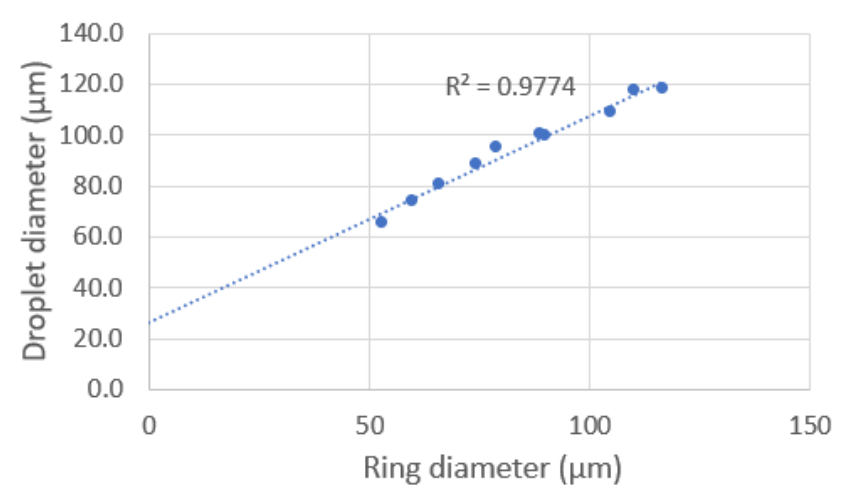

Fig. 8 Measurement results of the relation between the ring diameter and droplet diameter.

In the conventional LIFT method, the droplet size increases with the increasing irradiation energy [13, 20]. Using BR-LIFT, stable printing can be performed because the droplet diameter does not depend on the irradiation energy.

Figure 8 indicates the relation between the ring diameter and droplet diameter. This experiment was performed with the optimum pulse energy for each ring diameter. The pulse energies ranged from 18 to $39 \mu \mathrm{J}$.

The pulse energy increases with the increasing ring diameter. The dotted line shows the linear approximation of the measurement results. The squared value of the correlation coefficient had a large positive correlation with 0.9774. The droplet diameter exhibits extremely high linearity with respect to the ring diameter.

Once the ring diameter is determined, the droplet diameter can be determined under identical donor conditions. Thus, the droplet diameter can be controlled by changing the ring diameter. The droplet size is an important parameter directly related to the printing quality. In the LIFT system with a Gaussian beam, the droplet diameter cannot be easily controlled because the droplet diameter depends on the pulse energy. In contrast, in the BR-LIFT method, the droplet diameter can be controlled by changing the ring diameter. The controllability of the system can be improved because high-definition printing can be performed with a small ring diameter and high-speed printing can be performed with a large ring diameter.

\subsection{Possibility of long flight distance}

Figure 9 shows the landing droplets when the gap is changed to 200, 500, 700, and $1000 \mu \mathrm{m}$. The ring diameter was $115 \mu \mathrm{m}$, and the pulse energy was $37 \mu \mathrm{J}$. In case of LIFT with a Gaussian beam, the gap tolerance that satisfies the droplet quality was approximately $100 \mu \mathrm{m}$ at the longest [13, 37]. In contrast, the droplets maintain high quality even if the gap was increased to $1000 \mu \mathrm{m}$ in the BR-LIFT method.

$$
\begin{aligned}
\text { Gap } & =\mathbf{2 0 0} \\
\text { Gap } & =\mathbf{5 0 0}
\end{aligned}
$$

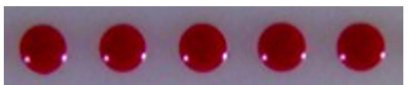

$$
\text { Gap }=700
$$

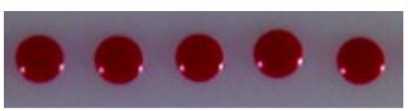

$$
\text { Gap }=\mathbf{1 0 0 0}
$$

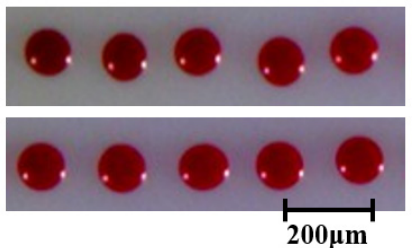

Fig. 9 Landing droplets when the gap is changed to 200, 500, 700, and $1000 \mu \mathrm{m}$.

Next, the gap was increased to $2000 \mu \mathrm{m}$. Figure 10 shows the relation between the gap distance and average droplet diameter at 32 measurement points. The ring diameter was $115 \mu \mathrm{m}$, and the pulse energy was $37 \mu \mathrm{J}$. The average droplet diameter was observed to be independent of the gap distance.

The variation tends to increase from $1.2 \%$ to $2.4 \%$ as the gap increases ten times from 200 to $2000 \mu \mathrm{m}$. However, the variation of $2.5 \%$ or less is comparable to that of electrophotography [35]. The generation of satellites may be observed when the gap is greater than $1000 \mu \mathrm{m}$. In this case, 
the optimization of laser parameters allows the formation of high-quality droplets.

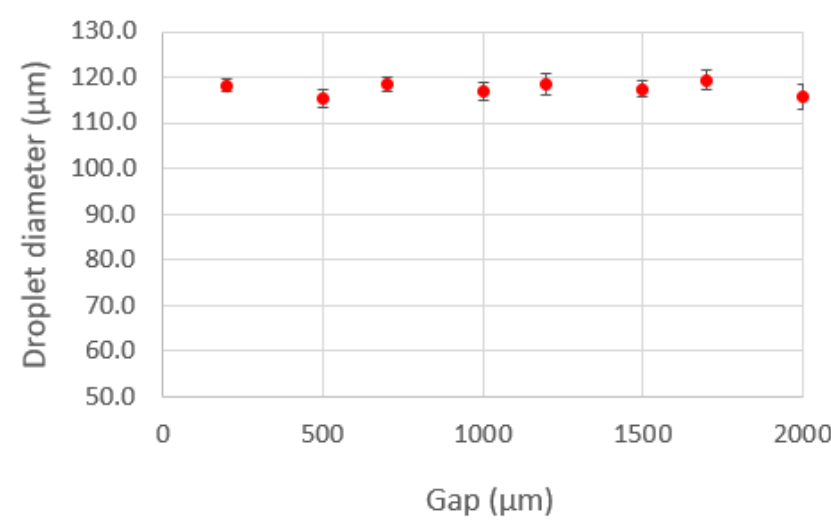

Fig. 10 Measurement results of the relation between the gap distance and average droplet diameter.

\subsection{QR code printing application}

We present QR code printing as an application example. The donor conditions were a viscosity of $4 \mathrm{~Pa} \cdot \mathrm{s}$ and a film thickness of $9 \mu \mathrm{m}$. The ring diameter was $110 \mu \mathrm{m}$, and the pulse energy was $26 \mu \mathrm{J}$. The experimental conditions included a scanning speed of $\mathrm{V}=200 \mathrm{~mm} / \mathrm{s}$, a gap of 500 $\mu \mathrm{m}$, and an overlap rate ( 1 - interval/diameter) of 0.09 . Figure 11 shows the QR code printing result (https://www.ricoh.co.jp/). The size of the QR code was approximately $15 \times 15 \mathrm{~mm}^{2}$. The printed QR code could be read by a commercially available smartphone. This result indicates that BR-LIFT allows high-quality printing of complex and arbitrary patterns, proving its suitability for various applications.

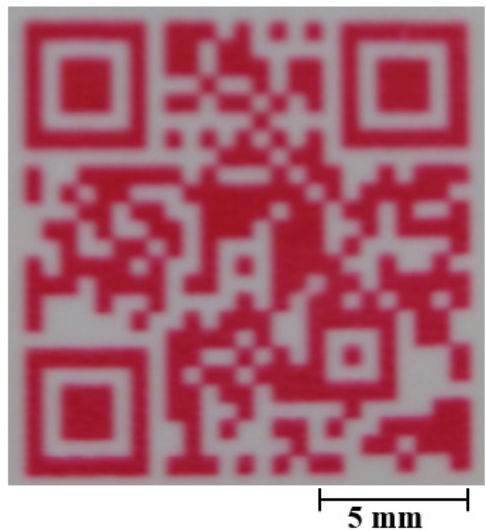

Fig. $11 \mathrm{QR}$ code printing (https://www.ricoh.co.jp/). The size of the QR code was approximately $15 \times 15 \mathrm{~mm}^{2}$.

\section{Conclusions}

In this study, we demonstrated the ejection of highviscosity inks by BR-LIFT under the pressure of a ringshaped vapor bubble (bubble ring or BR). BR creates an inward pressure toward the axis and wraps around the flying liquid. Therefore, BR-LIFT makes it possible to print high- quality droplets for long flight distances. The wavefront quality of the beam entering the beam converter plays an influential role with respect to the effectiveness of the BR. In particular, aberration correction of the incident beam is more important than beam conversion. The wavefront aberration was corrected using the double cylindrical lens method.

The landing droplet was microscopically observed to form an almost perfect circle under a gap of $500 \mu \mathrm{m}$. The droplet diameter is independent of the irradiation energy and dependent on the ring diameter. Based on the analysis, a dot size accuracy of $3 \%$ or less was obtained. This accuracy is comparable to that obtained in case of electrophotography. Further, the average droplet size was not dependent on the gap in the range of 200-2000 $\mu \mathrm{m}$. In addition, the printed QR code was readable by a commercially available smartphone. These results provide convincing evidence that BR-LIFT can be used in various practical applications, including security printing, bioprinting, and printed electronics.

\section{Acknowledgments}

The authors would like to thank Yoshio Wada for his valuable advice on the laser system and Keiichi Kato for his assistance with the laser prototype.

\section{References}

[1] H. J. J. Staat, A. Bos, M. Berg, H. Reinten, H. Wijshoff, M. Versluis and D. Lohse: Exp. Fluids, 58, (2017) 2.

[2] D. G. Foster: Int'l Symp. on Technol. for Digital Fulfillment 2009 (2009) 68.

[3] P. Delaporte, A. P. Alloncle: Opt. Laser Technol., 78, (2016) 33

[4] P. Serra and A. Piqué: Adv. Mater. Technol., 4, (2019) 1800099.

[5] I. Theodorakos, A. Kalaitzis, M. Makrygianni, A. Hatziapostolou, A. Kabla, S. Melamed, F. de la Vega, I. Zergioti: Adv. Eng. Mater., (2019) 21.

[6] A. Piqué, R.C.Y. Auyeung, K. M. Metkus, H. Kim, S. Mathews, T. Bailey, X. Chen and L.J. Young: SPIE Proc., 6879, (2008) 687911.

[7] Y. Chen, D. Munoz-Martin, M. Morales, C. Molpeceres, E. SánchezCortezon and J. Murillo-Gutierrez: Phys. Procedia, 83, (2016) 204.

[8] C. B. Arnold, P. Serra, and A. Piqué: MRS Bull, 32, (2007) 23.

[9] G. Hennig, T. Baldermann, C. Nussbaum, M. Rossier, A. Brockelt, L. Schuler and G. Hochstein: J. Laser Micro/Nanoengin., 7, (2012) 289.

[10] J Mikšys, G Arutinov, G Römer: Appl. Phys. A, 125, (2019) 814

[11] D. Munoz-Martina, C.F. Braszb, Y. Chena, M. Moralesa, C.B. Arnold and C. Molpeceres: Appl. Surf. Sci., 366, (2016) 389.

[12] V. Dinca, M. Farsari, D. Kafetzopoulos, A. Popescu, M. Dinescu and C. Fotakis: Thin Solid Films, 516, (2008) 6504.

[13] L. Rapp, J. Ailuno, A. P. Alloncle and P. Delaporte: Opt. Express, 19 , (2011) 21563

[14] E. Turkoz, L. Deike and C. B. Arnold: Appl. Phys. A, 123, (2017) 652

[15] E. Turkoz, A. Perazzo, H. Kim, H.A. Stone and C.B. Arnold: Phys. Rev. Lett., 120, (2018), 074501.

[16] E. Turkoz, S. Kang, L. Deike and C.B. Arnold: Phys. Rev. Fluids, 3 , (2018) 082201.

[17] R. Pohl: Ph.D. Thesis, University of Twente (2015). (Thesis)

[18] M. Zenou and Z. Kotler: Opt. Express, 24, (2016) 1431.

[19] P. Serra, M. Duocastella, JM Fernández-Pradas and J.L. Morenza: Appl. Surf. Sci., 255, (2009) 5342.

[20] M. Makrygianni, A. Millionis, C. Kryou, I. Trantakis, D. Poulikakos and I. Zergioti: Adv. Mater. Interfaces, 5, (2018) 1800440.

[21] H. Suhara, A. Tamura and T. Nishio: Proc. of LPM2018, (2018) \#18063.

[22] M. K. Bhuyan, et al.: Appl. Phys. Lett., 104, (2014) 021107.

[23] R. Nakamura, H. Kawaguchi, M. Iwata, A. Kaneko, R. Nagura, S. Kawano, K. Toyoda, K. Miyamoto and T. Omatsu: Opt. Express, 27, (2019) 38019

[24] K. Suzuki, M. Yoshino and T. Omatsu, Pat. No. WO2016/136722 (2016). (Patent) 
[25] M. S. Brown, N. T. Kattamis, and C. B. Arnold: Microfluid. Nanofluid., 11, (2011) 199.

[26] P. A. Belanger, M. Rioux: Appl. Opt., 17, (1978) 1080.

[27] M. Rioux, R. Tremblay, and P. A. Belanger: Appl. Opt., 17, (1978) 1532.

[28] K. Otomo, T. Hibi, Y. Kozawa, M. Kurihara, N. Hashimoto, H. Yokoyama, S. Sato, and T. Nemoto: Opt. Express, 22, (2014) 28215.

[29] J. Courtial and M.J. Padgett: Opt. Commun., 159, (1999) 13.

[30] K. Sueda, G. Miyaji, N. Miyanaga, and M. Nakatsuka: Opt. Express, 12, (2004) 3548.

[31] K. Toyoda, K. Miyamoto, N. Aoki, R. Morita and T. Omatsu: Nano Lett.,12, (2012) 3645.

[32] M. S. Brown, C. F. Brasz, Y. Ventikos and C. B. Arnold: J. Fluid. Mech., 709, (2012) 341.

[33] K. Yum and M. Yu: Nano lett., 6, (2006) 329

[34] R. E. Barrow and B. A. Hills: J. Physiol., 295, (1979) 217.

[35] P. Kajondecha, H. Cheng and Y. Hoshino: 2007 Int'l conf. on Digital Printing Technologies, (2007) 382.

[36] R. Nakamura, M. Iwata, A. Kaneko, K. Toyoda, K. Miyamoto and T. Omatsu: Proc. of CLEO2019, (2019) 1.

[37] M. Iwata et al.: Proc. of Imaging Conference JAPAN 2019 Fall Meeting, (2019) 197. (in Japanese)

[38] Sidney A. Self: Appl. Opt., 22, (1983), 658

[39] A. Patrascioiu, C. Florian, J. M. Fernandez-Pradas, J. L. Morenza, G. Hennig, P. Delaporte,3 and P. Serra: Appl. Phys. Lett., 105, (2014) 014101.

[40] A. Patrascioiu, J. M. Ferna'ndez-Pradas, A. Palla-Papavlu, J. L. Morenza and P. Serra: Microfluid Nanofluid, 16, (2014) 55.

[41] Y. L. Liu, Q. X. Wang, S. P. Wang, and A. M. Zhang: Phys. Fluids, $28,(2016) 122101$

(Received: June 5, 2020, Accepted: September 15, 2020) 